\title{
Digitalisierung, IT-Outsourcing, Informationssicherheit
}

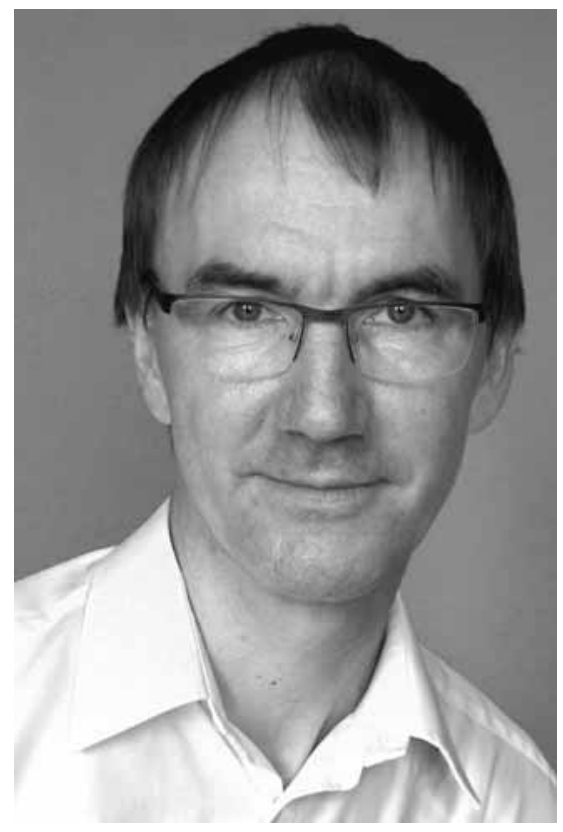

Die Optimierung der Geschäftsprozesse mit Hilfe moderner IT-Anwendungen hat alle Branchen erfasst. Dieser "Digitalisierung“ genannte Prozess steigert die Vernetzung zwischen den Produzenten und mit den Anwendern dramatisch. Hierbei spielen Netze, insbesondere das Internet, eine ganz wesentliche Rolle. Doch auch die IT entwickelt sich immer mehr zu einer Infrastruktur, denn die IT und die IT-Anwendungen können in dieser zweiten Hälfte des Informationszeitalters kaum mehr von den Anwenderunternehmen bereitgestellt und betrieben werden. IT/TK-Spezialisten übernehmen dies mehr und mehr. Anwenderunternehmen nutzen diese IT/TKDienste. In Ermangelung eines schöneren Begriffs nutzen wir den bekannten des "IT-Outsourcings".

Einen solchen Reifeprozess in Richtung stärkerer Spezialisierung und Arbeitsteilung haben andere Branchen bereits früher durchlaufen. Nun zieht die IT/TK nach. Dadurch entsteht ein Aufgabengebiet in der IT-Sicherheit, das zwar unterschwellig immer schon ein Thema war, aber bisher nicht konsequent genug angegangen wurde: Die IT-Sicherheit sucht immer noch mehr den Unternehmensbezug: Wertschöpfungsketten und Kundenbeziehungen werden weniger gut verstanden. Doch kennen wir die Antwort? Sind die IT-Sicherheitsspezialisten darauf vorbereitet, Informationssicherheit im Kontext von Lieferantennetzwerken zu denken und zu managen?

In diesem Heft widmen sich namhafte Experten folgenden Fragen: Was bedeutet die organisatorische Trennung zwischen dem IT-Hersteller/Betreiber auf der einen Seite und den Anwenderorganisationen ohne eigene IT/TK auf der anderen für die Informationssicherheit wirklich? Welche Anforderungen muss das IT-Sicherheitsmanagement in einer Welt der fortgeschrittenen Arbeitsteilung auf dem Gebiet der IT/ TK erfüllen? Welche Lücken bleiben bestehen und wie geht man mit den diesbezüglichen Risiken um? Was müssen Dienstleister tun, um Risikomanagement und Einflussnahme zu möglichen? Wie wird IT-Sicherheit bezahlbar? Inwieweit hemmen Standardisierung und Sicherheitsbedenken den Weg in die Cloud? Wie sollten Anwenderunternehmen mit Cloud-Risiken umgehen? Und wie können die Beteiligten in diesem Markt konkret zusammenarbeiten, um bestmögliche Sicherheit zu erreichen?

Wir wissen: IT/TK-Leistungen kann man "outsourcen“, aber das Risiko fürs Geschäft verbleibt primär beim Anwenderunternehmen. Dieses Heft zeigt die Herausforderungen und erläutert Methoden und Maßnahmen, um die Informationssicherheit im anbrechenden Zeitalter der "Digitalisierung" in allen Branchen adäquat und für beide Seiten (Dienstleister und Anwender) zufriedenstellend gewährleisten zu können. Wir fokussieren auf die Relationen im Business-to-Business (Behörden usw. einbegriffen) und betrachten Cloud-Anwendungen vor allem in diesem Bereich. Die Schnittstellen zum Endanwender (Consumer) und einfache Anwendungen für den privaten Gebrauch sind dabei nur sekundär. Im IT-Outsourcing, aber nicht nur dort, müssen diverse, das Sicherheits- und Service-Management betreffende Informationen verwaltet und ausgetauscht werden. Diesbezügliche Sicherheitsaspekte untersucht ein weiterer Aufsatz in diesem Heft.

Prof. Dr. Eberhard von Faber 